\title{
Earthquake Prediction: What should we be debating?
}

\section{ROBERT J. GELLER}

The topic posed by the editors for this debate, is whether the reliable prediction of individual earthquakes is a realistic scientific goal. Translated into everyday language, this becomes: given the present state of the art, does earthquake prediction research merit a significant investment of public funds? My initial contribution to this debate stated the negative case. None of the other debaters appears to have made strong arguments for the affirmative.

\section{Mission: Impossible?}

The arguments presented by some of the other debaters are variations on the following theme:

1. Prediction has not yet been shown to be impossible.

2. Some other things that were called impossible later turned out to be possible.

3. So, why shouldn't prediction also turn out to be possible?

However, convincing supporting data, not just the mere fact that impossibility has not yet been proven, should be required before any proposed research is approved. This is particularly true for fields like prediction 1 , or cold fusion 2 , where previous work has been notoriously unsuccessful. Note that we do not have to decide whether or not prediction is inherently impossible. We just have to decide whether or not there are compelling grounds at present for establishing large-scale ("throw money at the problem") programmes for prediction research. The answer given current knowledge is clearly negative, but the question could, if necessary, be reopened at any time if new proposals were backed by well documented and convincing results.

\section{The Gambler's Fallacy}

Simple mechanical systems used in games of chance provide cautionary examples for prediction researchers. If an honest die is rolled, the probability of each number's landing face up is 1 in 6 , but (due to sensitivity to small variations in the initial conditions) it is impossible to reliably and accurately predict the outcome of individual rolls.

Many gamblers nevertheless try in vain to look for patterns in the outcome of previous rolls of a die. Such gamblers, as a group, lose their money, but for a short time a lucky few will win. By looking only at the winners, while ignoring the much larger group of losers, it is easy to jump to the mistaken conclusion that the winners have found a way to beat the odds.

The root cause of the gambler's fallacy is drawing conclusions froma retrospectively chosen and unrepresentative sample of a much larger data set. This is also the fundamental problem bedevilling 'case studies' of alleged earthquake precursors, but the fallacy here is a bit less obvious. This is because the probabilities for each roll of a die are fixed, but the probability of earthquake occurrence is spatially variable, and varies strongly temporally depending on previous seismicity.

\section{A benchmark for prediction methods}

The probability of earthquake occurrence is much larger than usual immediately after an earthquake occurs, decaying with time as a power law ${ }^{3}$. This is the basis for the 'automatic alarm' prediction strategy 4 : issue an alarm 
automatically after every earthquake above a certain size, on the chance that it might be a foreshock of a larger earthquake.

The exact success and alarm rates of the automatic alarm strategy will depend on the choice of windows, but there will probably be hundreds of false alarms for every success, and on the order of half the significant earthquakes will probably be missed. Thus, as emphasized by its proposer ${ }^{4}$, this strategy is not in general sufficiently reliable and accurate to justify issuing public alarms.

(Probabilistic prediction of aftershocks -- see discussion by Michael -- may be an exception where public alarms are justifiable.) Note that the 'automatic alarm' strategy is a scientifically valid method for making forecasts in Main's category 2 (time-dependent hazard), although its actual utility in hazard mitigation is unclear.

The automatic alarm strategy can be implemented at essentially no cost, as all we need are the hypocentral data from a seismic network. No measurements of electromagnetic signals, radio-isotope levels in well water, or any of the other phenomena that are sometimes claimed to be earthquake precursors are required. Although the automatic alarm strategy falls far short of the accuracy and reliability required for issuing public alarms, it achieves a significant probability gain over predictions issued completely at random. The automatic alarm strategy should be adopted as the benchmark for testing other proposed prediction methods. Unless and until a proposed method has been shown to outperform the automatic alarm strategy (none has ever been shown to do so), it does not warrant intensive investigation.

\section{Needed: objective testing, not case studies}

What is wrong with present prediction research? Wyss cites scientifically weak work and scientifically unqualified publicity seekers as problems. I agree $^{5}$, but I do not think these are the main problems. The principal problem appears to be the use of the anecdotal 'case study' approach by prediction researchers. At an early stage this approach can be valuable, but there are now literally thousands of published claims of precursors ${ }^{1}$. The value of further additions to this list is questionable.

Wyss's contribution to this debate cites both "increased moment release" (more small earthquakes than usual) and "seismic quiescence" (fewer small earthquakes than usual) as precursors. Thus it appears that any variability whatsoever in background seismicity can be claimed, after the fact, to have been a precursor. To determine whether these variations in seismicity levels are random fluctuations or real physical phenomena, objective testing of unambiguously stated hypotheses is required 6 .

It is regrettable that the other contributors to the first two weeks of this debate have not sufficiently acknowledged the importance of objective statistical testing in resolving the prediction debate. Researchers looking for precursors could greatly benefit from the experience of pharmaceutical research, where new drugs are routinely evaluated by randomized double-blind testing using placebos $^{7}$.

\section{Long-term forecasts: where do we stand?}

If they were reliable and accurate, long-term forecasts could be useful in engineering and emergency planning measures to mitigate the impact of earthquakes. Unfortunately, however, there is serious question about the accuracy and reliability of proposed methods for long-term seismicity forecasts. For example, several long-term forecasts have been issued on the basis of the 'seismic gap' hypothesis. However, when these forecasts were later subjected to independent testing, they were shown not to have outperformed random chance ${ }^{8}$. There has been a running controversy over the seismic-gap forecasts; for information on both sides see the works cited in the bibliography of ref. 8 . 
term forecasts were for an earthquake on a different fault, and with a different focal mechanism, than the actual earthquake (see section 4.4 of ref. 1).

Furthermore, even if the claim of 'success' were warranted, this appears to be a classic example of the gambler's fallacy of picking one possibly atypical sample out of a much larger dataset.

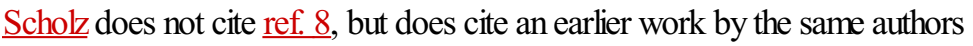
in his discussion of the seismic-gap hypothesis. Scholz contends that Kagan \& Jackson incorrectly rejected the seismic gap hypothesis because their study considered some earthquakes that were too small. Scholz's criticism is apparently based on ref. 9, but Jackson \& Kagan replied to this criticism in ref. 10. Unfortunately Scholz discussed only the criticism but not the reply.

It appears that the real problem is that the original forecasts did not fully specify the 'rules of the game', thus forcing anyone who evaluates these forecasts to choose some of the rules in retrospect, after the actual seismicity is already known. (In fairness, it must be added that the forecasts in question are among the best of their kind, as they were stated with sufficient precision to be objectively tested, albeit with some ambiguity.) The only way to avoid such problems in the future is for forecasters and independent evaluators to thoroughly thrash out all of the ground rules at the time a forecast is issued, before the actual seismicity is known.

\section{The downside of long-term forecasts}

Until we have well validated methods, we should be reluctant to recommend that government authorities take strong action on the basis of long-term forecasts, although no great harm and some good is likely to result from taking sensible precautionary measures on a moderate scale in regions for which long-term forecasts have been issued.

There is, however, a risk that the authorities in regions for which long-term forecasts have not been issued may become overly complacent. This is not merely a theoretical possibility. Several hypothetical future earthquakes in and around the Tokyo area have been the subject of extensive discussion in Japan for the past 25 years (see section 5 of ref. 1). Partly as a result of these forecasts, local governments in western Japan, including Kobe, incorrectly assumed that their region was not at significant risk, and failed to take sufficient precautionary measures against earthquakes. This was one of the causes of the unexpectedly large damage caused by the 1995 Kobe earthquake.

\section{The bottom line}

Rather than debating whether or not reliable and accurate earthquake prediction is possible, we should instead be debating the extent to which earthquake occurrence is stochastic. Since it appears likely that earthquake occurrence is at least partly stochastic (or effectively stochastic), efforts at achieving deterministic prediction seem unwarranted.

We should instead be searching for reliable statistical methods for quantifying the probability of earthquake occurrence as a function of space, time, earthquake size, and previous seismicity. The case study approach to earthquake prediction research should be abandoned in favour of the objective testing of unambiguously formulated hypotheses. In view of the lack of proven forecasting methods, scientists should exercise caution in issuing public warnings regarding future seismic hazards. Finally, prediction proponents should refrain from using the argument that prediction has not yet been proven to be impossible as justification for prediction research.

\section{Robert J. Geller}

Department of Earth and Planetary Physics, Graduate School of Science,

Tokyo University,

Bunkyo, Tokyo 113-0033,

Japan.

bob@global.geoph.s.u-tokyo.ac.jp 


\section{References}

1. Geller, R.J. Earthquake prediction: a critical review. Geophys. J. Int. 131, 425-450 (1997).

2. Huizenga, J.R. Cold Fusion: The Scientific Fiasco of the Century (University of Rochester Press, Rochester, NY, 1992).

3. Kagan, Y. \& Knopoff, L. Statistical short-term earthquake prediction. Science 236, 1563-1567 (1987).

4. Kagan, Y. VAN earthquake predictions: an attempt at statistical evaluation. Geophys. Res. Lett. 23, 1315-1318 (1996).

5. Geller, R.J. Predictable publicity. Astron. Geophys Quart. J. R. Astr. Soc. 38(1), 16-18 (1997).

6. Jackson, D.D. Hypothesis testing and earthquake prediction. Proc. Natl Acad. Sci. USA 93, 3772-3775 (1996).

7. Shapiro, A.K. \& Shapiro, E. The powerful placebo (Johns Hopkins University Press, Baltimore, MD, 1997).

8. Kagan, Y.Y. \& Jackson, D.D. New seismic gap hypothesis: Five years after. J. Geophys. Res. 100, 3943-3959 (1995).

9. Nishenko, S.P. \& Sykes, L.R. Comment on "Seismic Gap Hypothesis: Ten years after" by Y.Y. Kagan and D.D. Jackson. J. Geophys. Res. 98, 9909-9916 (1993).

10. Jackson, D.D. \& Kagan, Y.Y. Reply. J. Geophys. Res. 98, 99179920 (1993).

Nature@ Macmillan Publishers Ltd 1999 Registered No. 785998 England. 